\section{Split world}

\section{Decoherence and the Quantum-to- \\ Classical Transition \\ by Maximilian Schlosshauer \\ Springer: 2007.416 pp. $€ 54$}

\section{Anton Zeilinger}

Elementary particles, such as photons, electrons and neutrons inhabit a quantum world, where they may exist in a superposition between two or more locations at the same time. They can be entangled over large distances, or several of their properties may be undefined. In our everyday world, such phenomena are not evident - computers, cars and people are always at well-defined locations. And the properties of one are independent of what is done to another one at a distant location. Why do we not see quantum phenomena in macroscopic objects?

The term 'decoherence' describes how quantum superposition is lost as a result of entanglement between an environment and the internal or external degrees of freedom of the system. Whether or not this can explain the emergence of a classical world is a matter of philosophical debate. Nonetheless, the physics of decoherence is interesting. Maximilian Schlosshauer gives us a thorough and readable representation of today's understanding of the topic in Decoherence and the Quantum-to-Classical Transition.

The book gives an excellent overview of the various theoretical approaches to the physics that leads to decoherence. A particular strength is that it includes accounts of several experiments demonstrating the decoherence mechanism in detail, and shows how the results might predict experimental developments with quantum systems that become larger and more complex.

Schlosshauer also discusses various approaches to the fundamental and conceptual understanding of the nature of quantum

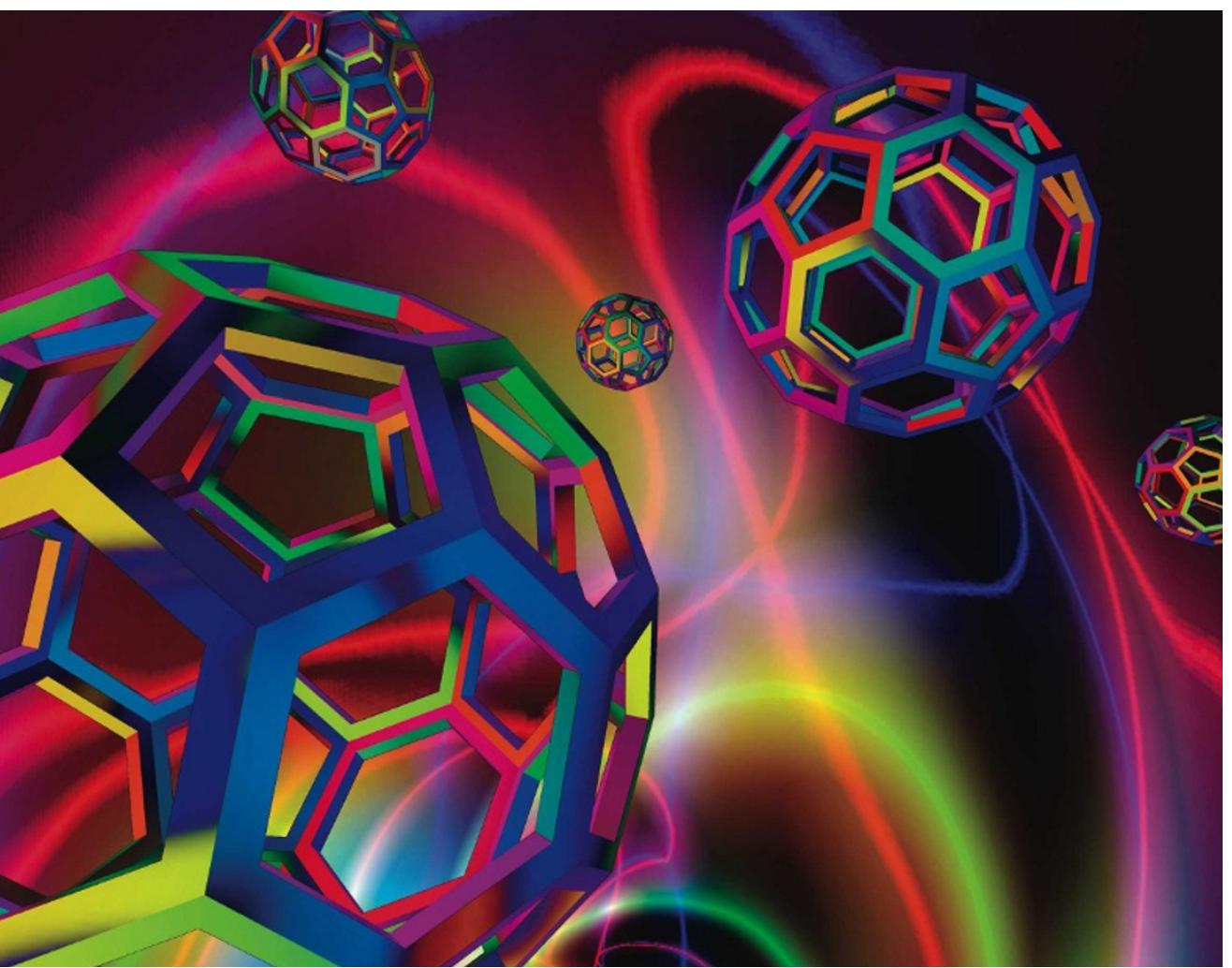

Double life: these carbon- 60 fullerenes can behave as either a quantum or a classical system. states, and therefore of decoherence. Although he maintains a neutral position, I have the impression that he is taking the notion of quantum states in an ontological rather than an epistemological sense. For example (as is well known from early quantum mechanics and is now supported by experiment), there is no fixed boundary between the classical and the quantum world. The same object can behave as a quantum system in one situation, for example when it is isolated from the environment, or as a classical system in another.

Fullerenes and even biological macromolecules are typical examples, showing quantum interference in two-slit experiments whereas they can be seen in a tunnelling electron microscope, for instance, at classically welldefined locations. This shifting boundary is confirmed by the decoherence mechanism. But to argue that this is evidence against the Copenhagen interpretation, as the author does, is unjustified: the Copenhagen interpretation itself says that whether an object is classical or quantum is a function of the chosen experimental set-up.

Decoherence is, to follow physicist John Bell, for all practical purposes sufficient to describe the loss of quantum features for large systems. There are still unanswered questions. It is well known, which Schlosshauer also stresses, that the interference terms never strictly vanish, so decoherence can tell us only that the interference terms disappear effectively but not rigorously. Even after accepting that approximation, we are still left with the system represented as a mixture of various possibilities, like being in two places at once. In the classical world, we know that the system is always at this place or at that place. To explain the two as equivalent is again, for all practical purposes, sufficient. Yet it involves, as Bell points out, another interpretive leap.

An author index and more discussion of the early history of decoherence would have been useful, but the book is an important resource for anyone interested in decoherence. It is very well written and it will contribute to further conceptual and theoretical development and to new experiments, extending the boundary of the quantum domain - maybe even into the macroscopic world.

Anton Zeilinger is professor of physics at the University of Vienna and the Austrian Academy of Sciences in Vienna, Austria.

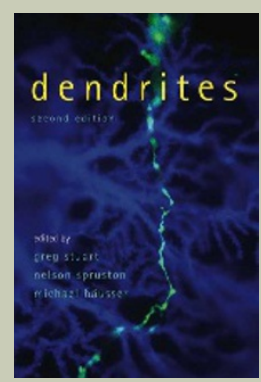

Dendrites

edited by G. Stuart, N. Spruston and Michael Häusser (Oxford Univ. Press: 2007. 2nd edition. $€ 42.95, \$ 79.50)$ Dendrites are extensions of the cell body of the neuron, receiving synaptic input from thousands of other neurons and shaping and integrating these signals. This book, which is a collection of informative essays by prominent neuroscientists, captures the resurgence of interest in these structures, the intricacies of their function, and the contribution they make to mental processes.

The second edition follows eight years after the first.
Chapters have been extensively revised or rewritten, and six of the 20 are new - reflecting the vigorous expansion of the topic into new areas. A multidisciplinary approach underpins the search for answers to the many questions posed by dendrites and gives insight into their development and their electrical, chemical and computational properties.

Each chapter is lavishly referenced and set out under concise subheadings; the colour illustrations, a staple of any worthwhile learning aid, are thoughtfully presented. There is a foreword by Nobel laureate Bert Sakmann. 